\title{
Identification of Triptophenolide from Tripterygium wilfordii as a Pan- antagonist of Androgen Receptor
}

\author{
Yang He, Meng Wu, Yangguang Liu, Quanjie Li, Xiaoyu Li, Laixing Hu,* Shan Cen,* and Jinming Zhou* \\ Institute of Medicinal Biotechnology, Chinese Academy of Medical Science, Beijing, China
}

Supporting Information

ABSTRACT: A compound, triptophenolide, derived from Tripterygium wilfordii was identified as an antiandrogen. Triptophenolide inhibits the activity of both wild-type and F876L mutant androgen receptors. Triptophenolide exhibits its antiandrogenic activity through competitive binding with androgen in the hormone-binding pocket, decreasing the expression of androgen receptor, and reducing the nuclear translocation of androgen receptor.
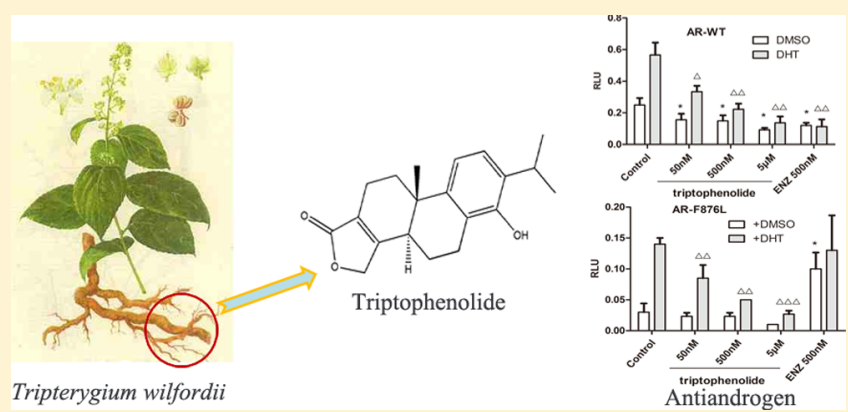

KEYWORDS: Androgen receptor, triptophenolide, gene report system, traditional Chinese medicine
$\mathrm{P}$ rostate cancer $(\mathrm{PCa})$ is one of the most common cancers and the second leading cause of cancer death in men of the western countries. ${ }^{1}$ Androgen receptor (AR) plays a critical role in the development and progression of $\mathrm{PCa} .{ }^{2}$ Antiandrogen has become a main therapeutic agent for advanced PCa. Up to now, current clinically used antiandrogens such as flutamide (FLU), bicalutamide (BIC), and newly approved enzalutamide (ENZ) mainly target the hormone-binding pocket (HBP) of the AR ligand binding domain (LBD). These approved antiandrogens have greatly improved the survival and life quality of the PCa patients. However, after the initial effective response, most tumors develop resistance to the antiandrogen used for therapy due to the AR overexpression, ${ }^{3}$ the AR mutants, ${ }^{4-7}$ the bypassing pathway activation, ${ }^{8,9}$ etc. Therefore, it is increasingly urgent to seek for the new strategy to obtain novel antiandrogens in order to circumvent the developed resistance of PCa for the current clinically used antiandrogens. However, traditional Chinese medicine (TCM) has a very long history in cancer therapy. To date, TCM is widely used as a complementary therapy for cancer and is found improving symptoms and the quality of life and even extend lifespan of cancer patients, ${ }^{10,11}$ thus providing a potential resource for therapeutic agents of prostate cancer. Importantly, one herb, Tripterygium wilfordii, apart from its application in the treatment of rheumatoid arthritis and other immune-mediated inflammatory diseases for the anti-inflamatory and immunomodulatory activity, ${ }^{12,13}$ is also very frequently used in therapy of cancer in Chinese hospitals. Some natural products derived from the herb, such as celastrol ${ }^{14,15}$ and triptolide, ${ }^{16,17}$ were reported as potent anti-PCa agents. What is more, through a genomics-based phenotypic screening approach, triptophenolide was found as an effective inhibitor of $\mathrm{AR}^{18}$ however, the detailed antiandrogen mechanism remains unknown. ${ }^{18}$ Herein, starting from the detection of the antiandrogen activity of ethanol extract of Tripterygium wilfordii root, we finally identified triptophenolide as a pan-antagonist for wild-type and mutant ARs, which inhibits androgenic activity through competitive binding with androgen in the $\mathrm{HBP}$ of $\mathrm{AR}$, decreasing the expression of androgen receptor and reducing the nuclear translocation of AR.

The strategy is shown in Scheme 1. We tried to screen the antiandrogen from Tripterygium wilfordii through combining

Scheme 1. Strategy Used in Search of Antiandrogen Derived from Tripterygium wilfordii

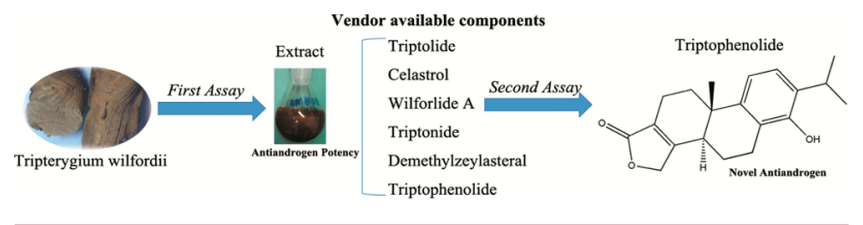

MTT assay and dual-luciferase assay. As a start, we inspected the antiandrogenic potency of Tripterygium wilfordii root ethanol extract through MTT and dual-luciferase assay. In MTT assay, both LNCaP (AR positive) and PC-3 (AR negative) cell lines were used to evaluate the antiprostate cancer activity of the herb. As a result, Tripterygium wilfordii root extract inhibited the growth of the LNCaP cells rather than the PC-3 cells in a significant dose-dependent manner at the concentration range of $1.0-7.5 \mu \mathrm{g} / \mathrm{mL}$ (Figure S1A). Such difference in cell growth inhibition between the LNCaP cells and the PC-3 cells of the extract suggested its inhibition of the AR activity, thus making the LNCaP cells more sensitive.

Received: April 30, 2016

Accepted: September 28, 2016

Published: September 28, 2016 
Following dual-luciferase assay of AR activity in LNCaP cells confirmed that Tripterygium wilfordii root extract significantly inhibited the AR transcription activity at the concentration range of $5.0-7.5 \mu \mathrm{g} / \mathrm{mL}$ (Figure S1B), which elucidated the more sensitivity of LNCaP cells against the extract.

To inspect which components in Tripterygium wilfordii root should be responsible for the antiandrogen activity, we tested six main components from Tripterygium wilfordii, and the results were shown in Figure S2. The luciferase assay of the AR activity in LNCaP cells revealed that all six components could significantly reduce the endogenous $\mathrm{AR}$ transcription activity (Figure S2A) at the concentration of $5 \mu \mathrm{M}$, and triptophenolide, triptolide, and triptonide displays significant anti-AR activity potency at the concentration of $500 \mathrm{nM}$. As AR mutant F876L will cause the drug resistance of the clinical used antiandrogen $\mathrm{ENZ},{ }^{19}$ we further investigated the antiandrogenic activities of all these six components against the F876L mutant AR. AR-dependent reporter assays were performed in PC-3 cells using ARE (androgen receptor element) driven PSAluc reporter and AR-expressing plasmids. Antiandrogen ENZ was included as the control. As a result, in the absence of dihydrotestosterone (DHT), the F876L mutated AR was activated by ENZ (Figure S2B), which was consistent with previous reports. ${ }^{20}$ Only triptophenolide demonstrates the significant antiandrogenic activity in suppressing DHT-induced transcription of the endogenous F876L mutated AR at the concentration of $500 \mathrm{nM}$ (Figure S2B). Thus, we selected triptophenolide as a potential antiandrogen for further mechanism studies.

To further evaluate the antiandrogenic potency of triptophenolide, we investigated antiandrogenic activity of triptophenolide against wild-type (WT) and T877A (FLU resistance mutant), F876L, and W741C + T877A (FLU and $\mathrm{BIC}$ resistance mutant) mutated ARs in absence or presence of DHT at $50 \mathrm{nM}, 500 \mathrm{nM}$, and $5 \mu \mathrm{M}$. As results, triptophenolide did not show any agonistic activity to both the wild-type and mutated ARs in absence of DHT (Figure 1A-D, white bars). In presence of DHT, triptophenolide demonstrates effective antiandrogenic activity in suppressing DHT-induced transcriptions of the WT, F876L, T877A, and W741C + T877A AR mutants in a dose-dependent manner Figure $1 \mathrm{~A}-\mathrm{D}$, black bars), which indicates triptophenolide is a pan-antagonist against WT and mutated ARs (the results of the luciferase assay of the ARs activity for triptophenolide within the $0.1-25 \mu \mathrm{M}$ concentration range are listed in Figure S3). We also evaluated the $\mathrm{IC}_{50}$ value of triptophenolide against $\mathrm{AR}$ transcription activity. The result showed that triptophenolide has the $\mathrm{IC}_{50}$ values of $260 \mathrm{nM}$ for AR-WT, $480 \mathrm{nM}$ for AR-F876L, $388 \mathrm{nM}$ for AR-T877A, and $437 \mathrm{nM}$ for W741C + T877A (Figure S4), respectively. Next, the regulation of PSA at RNA level by triptophenolide was detected using RT-PCR in LNCaP cells. The results showed that triptophenolide significantly downregulated PSA mRNA level at $500 \mathrm{nM}$ and $5 \mu \mathrm{M}$ (Figure 1E, the measured value of RT-PCR assay were listed in Table S3). It means that the inhibition of the PSA expression by triptophenolide is modulated at mRNA level. Next, the in vitro cytotoxicity of triptophenolide was evaluated by MTT assays in LNCaP and PC-3 cells. As a result, triptophenolide inhibits the growth of the LNCaP cells rather than the PC-3 cells in a significant dose-dependent manner at the concentration range of $0-5.0 \mu \mathrm{M}$ (Figure $1 \mathrm{~F}$ ). Such difference in cell growth inhibition between the LNCaP cells and the PC-3 cells
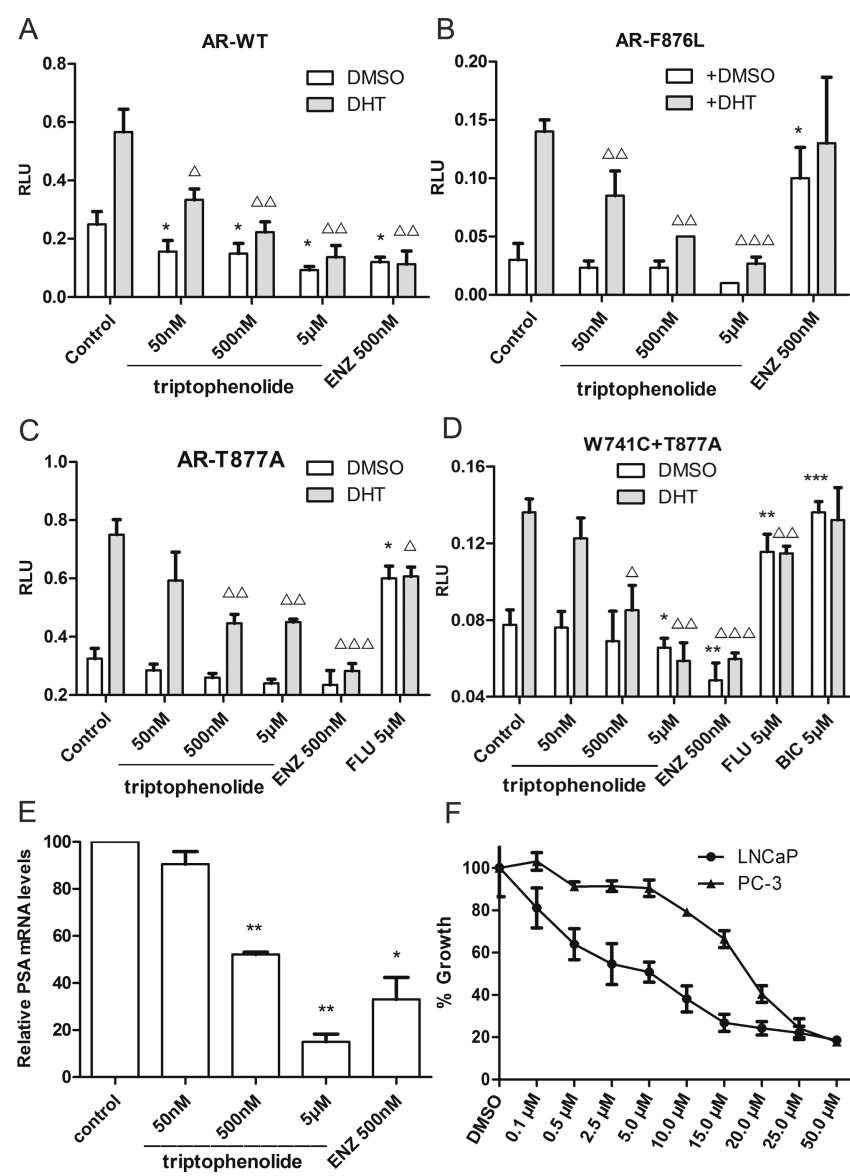

$\mathrm{F}$

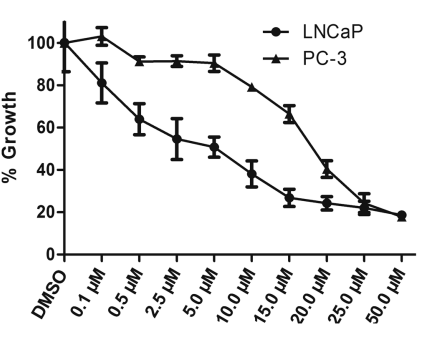

Figure 1. Triptophenolide is identified as an antiandrogen: (A) Effect of triptophenolide on wild-type AR activity in PC-3 cells. (B) Effect of triptophenolide on F876L AR activity in PC-3 cells. The F876L mutated AR was activated by ENZ at $500 \mathrm{nM}$. (C) Effect of triptophenolide on T877A AR activity in PC-3 cells. The T877A mutated AR was activated by hydroxyflutamide at $5 \mu \mathrm{M}$. (D) Effect of triptophenolide on T877A+W741C AR activity in PC-3 cells. The T877A + W741C mutated AR was activated by both hydroxyflutamide and bicalutamide at $5 \mu \mathrm{M}$. (E) Triptophenolide significantly downregulated PSA mRNA level at $500 \mathrm{nM}$ and $5 \mu \mathrm{M}$. (F) Growth inhibitory effects of triptophenolide in the PC-3 and LNCaP cells. Plasmids expressing ARs are transiently transfected in PC-3 cells in dual luciferase assay. Experiments were in triplicate. ${ }^{*} P<0.05$, $* * P<$ $0.01, * * * P<0.001$ vs DMSO group, $\triangle P<0.05, \triangle \triangle P<0.01, \triangle \triangle \triangle$ $P<0.001$ vs DHT group. BIC, bicalutamide; ENZ, enzalutamide; $\mathrm{DHT}$, dihydrotestosterone. All results are shown as mean $\pm \mathrm{SD}$.

of triptophenolide might be caused by its antiandrogenic activity.

To investigate whether triptophenolide exhibits its antiandrogenic activity through competitive binding with androgen in the HBP, the AR fluorescence polarization (FP) assay was performed with PolarScreen AR competitor assay kit (P3018, Invitrogen). When the tracer is free in solution, its rotational mobility is greater than when bound to the receptor, resulting in a low fluorescence polarization value. We have controlled the assay for minimal competition (DMSO vehicle), which has a maximum value of fluorescence polarization and for no receptor (tracer only), which represents the minimum value of the fluorescence polarization that can possibly be reached by a competitor. The ENZ was included as a positive control (Figure 2). Triptophenolide reduced the fluorescence polarization value in a dose-dependent manner at the concentration 

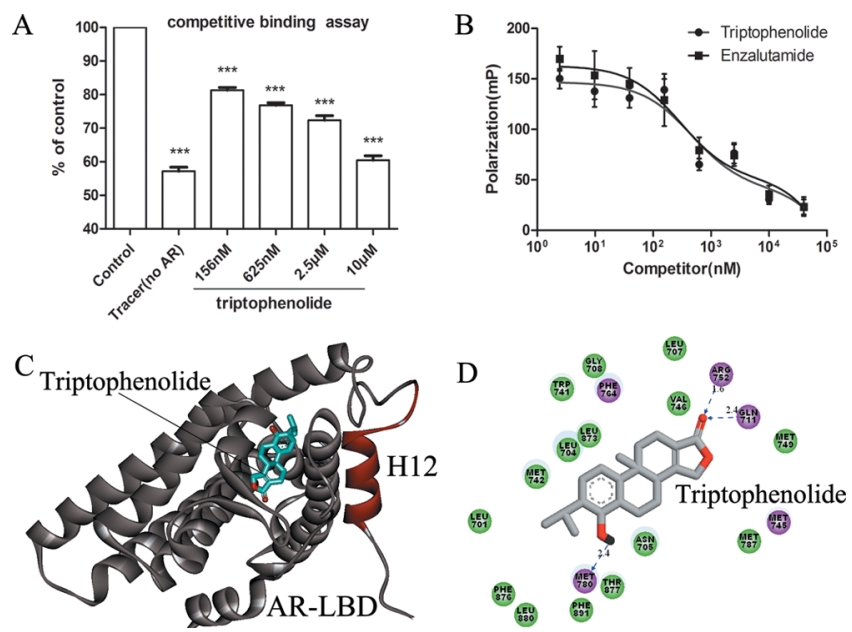

Figure 2. (A) Competitive binding of triptophenolide to the AR-LBD evaluated by AR fluorescence polarization (FP) assay. (B) The inhibition curves of triptophenolide and ENZ. (C) Triptophenolide (in stick, carbon in gray) fits the HBP of AR quit well in the predicted binding mode. (D) The detail interactions between triptophenolide and $\mathrm{AR}$, hydrogen bond is indicated by the black dashed line with arrow. Experiments were in triplicate. ${ }^{*} P<0.05$, ${ }^{* *} P<0.01$, *** $P<$ 0.001 vs control group. All results are shown as mean \pm SD.

range of $10 \mathrm{nM}$ to $10 \mu \mathrm{M}$, indicating it binds to AR-LBD by competing with the tracer over the HBP Figure $2 \mathrm{~A}$ ). The $\mathrm{IC}_{50}$ value computed from the inhibition curve of triptophenolide was $467 \mathrm{nM}$ (Figure 2B), comparable to ENZ (294 nM, Figure 2B), which indicated that triptophenolide most probably exhibits its antiandrogenic activity via competitive binding with androgen in the HBP.

Next, we predicted the binding mode of triptophenolide in AR HBP through molecular docking using Molecular Operating Environment (MOE) version 2009.10. To date, crystal structure of AR-LBD at the antagonistic form has not been obtained. Thus, we built a structural model of the WT AR-LBD with $\mathrm{H} 12$ at the antagonistic form, using crystal structures of the AR-LBD/DHT and ER/antagonist complexes (PDB entries: 1t65 and 3ert) as templates. Three other antiandrogens (ENZ, BIC, FLU) were also docked as reference compounds (Figure S5), and triptophenolide had the second most favorable docked score after ENZ (Table S4). The predicted binding model of triptophenolide in the antagonistic model of WT AR-LBD was shown as Figure 2C,D. Triptophenolide fits the HBP of AR quite well (Figure 2C), and it is assumed to form hydrogen bond interactions with Gln711, Arg752, and Met780 as well as the hydrophobic interactions with Leu701, Leu704, Leu707, Met742, Met745, Met749, Ph4764, Met780, Leu873, and Phe876 at the HBP (Figure 2D), which indicates that triptophenolide should bind at the HBP in a high specificity.

To determine whether triptophenolide would modulate AR protein expression, we further examined the AR protein levels during triptophenolide treatment. Western blot analysis indicated triptophenolide effectively suppressed $A R$ protein expression in LNCaP cells in a dose-dependent manner at 50 $\mathrm{nM}, 500 \mathrm{nM}$, and $5 \mu \mathrm{M}$ for $24 \mathrm{~h}$ (Figure $3 \mathrm{~A}$ ). Thus, besides competitive binding with androgen in HBP, triptophenolide is also able to exert the antiandrogen potency through the downregulation of the AR protein level. Upon activation by androgen, the AR translocates into the nucleus where it binds ARE sites of DNA. Therefore, the confocal assay was done to
A

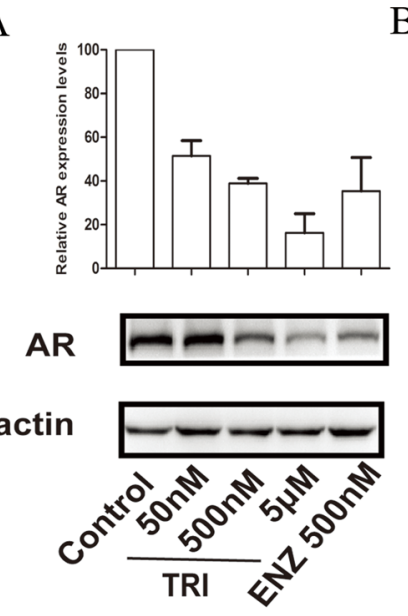

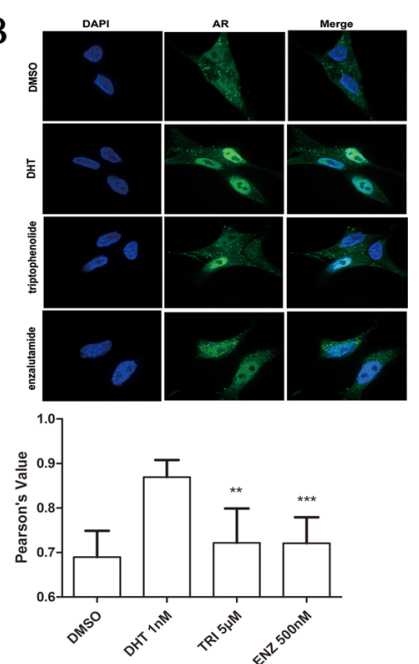

Figure 3. (A) Western blot analysis indicates that triptophenolide suppresses AR protein expression in LNCaP cells at $500 \mathrm{nM}$ and 5 $\mu \mathrm{M}$. (B) Confocal assay indicates triptophenolide efficiently inhibits DHT induced AR nuclear translocation. Experiments were in triplicate. $* P<0.05$, $* * P<0.01, * * * P<0.001$ vs control group. TRI, triptophenolide. All results are shown as mean \pm SD.

evaluate the $\mathrm{AR}$ nuclear translocation in the presence of triptophenolide. As a result, in the presence of $1 \mathrm{nM} \mathrm{DHT}$, the ratio of nuclear versus cytoplasmic $\mathrm{AR}$ in triptophenolide (5 $\mu \mathrm{M})$ treated cells was comparable to ENZ $(500 \mathrm{nM})$ treated cell, 3-fold reduced relative to DHT alone (Figure 3B). It indicates that triptophenolide is able to inhibit DHT induced AR nuclear translocation and further confirms that triptophenolide is a bona fide antiandrogen.

Here, we first discovered that the TCM herb Tripterygium wilfordii could efficiently inhibit the AR transcription activity. Further we identified triptophenolide from Tripterygium wilfordii as a pan-antagonist for wild-type and mutant ARs. Specially, triptophenolide has high binding affinity with AR and inhibits the activity of F876L mutant AR, which turns ENZ from $\mathrm{AR}$ antagonist to agonist. In addition, triptophenolide could reduce the $\mathrm{AR}$ protein levels. Another resistance mechanism of ENZ during the treatment of $\mathrm{PCa}$ is the activation of the glucocorticoid receptor (GR) signaling. ${ }^{8}$ It has been reported that triptophenolide is also an inhibitor of $\mathrm{GR},{ }^{21}$ which could overcome the resistance caused by the bypassing pathway activation of GR. Thus, triptophenolide may offer an interesting anti-PCa lead compound for the further optimization. Moreover, the work further supports that the TCM is able to provide a potential resource of therapeutic agents for $\mathrm{PCa}$, and our strategy used here could also be extended to other TCM herbs to search for novel antiandrogens or other antiPCa compounds.

\section{ASSOCIATED CONTENT}

\section{Supporting Information}

The Supporting Information is available free of charge on the ACS Publications website at DOI: 10.1021/acsmedchemlett.6b00180.

NMR and MS analyses, biological assays details, and molecular modeling methods (PDF) 


\section{AUTHOR INFORMATION}

\section{Corresponding Authors}

*E-mail: zhoujinming@imb.pumc.edu.cn. Tel: 86-1063131011. Fax: 86-10-63017302.

*E-mail: shancen@imb.pumc.edu.cn.

*E-mail: hulaixing@hotmail.com.

\section{Author Contributions}

The manuscript was written through contributions of all authors.

\section{Funding}

This work was in part supported by the Nature Science Foundation of China (81311120299, 81271844, 81672559).

\section{Notes}

The authors declare no competing financial interest.

\section{ACKNOWLEDGMENTS}

We thank Dr. Jianhui Wu (Mcgill University) and Dr. Hiroyuki Seimilya (Cancer Chemotherapy Center, Japan) for providing AR expressing plasmids.

\section{ABBREVIATIONS}

$\mathrm{AR}$, androgen receptor; $\mathrm{ER}$, estrogen receptor; WT, wild-type; MTT, 3-(4,5-dimethyl-2-thiazolyl)-2,5-diphenyl-2H-tetrazolium bromide; PSA, prostate specific antigen; TCM, traditional Chinese medicine; LBD, ligand bind domain

\section{REFERENCES}

(1) Siegel, R.; Ma, J.; Zou, Z.; Jemal, A. Cancer statistics, 2014. CaCancer J. Clin. 2014, 64, 64.

(2) Dong, Y.; Zhang, H.; Gao, A. C.; Marshall, J. R.; Ip, C. Androgen receptor signaling intensity is a key factor in determining the sensitivity of prostate cancer cells to selenium inhibition of growth and cancerspecific biomarkers. Mol. Cancer Ther. 2005, 4, 1047.

(3) Scher, H. I.; Sawyers, C. L. Biology of progressive, castrationresistant prostate cancer: directed therapies targeting the androgenreceptor signaling axis. J. Clin. Oncol. 2005, 23, 8523.

(4) Korpal, M.; Korn, J. M.; Gao, X.; Rakiec, D. P.; Ruddy, D. A.; Doshi, S.; Yuan, J.; Kovats, S. G.; Kim, S.; Cooke, V. G.; Monahan, J. E.; Stegmeier, F.; Roberts, T. M.; Sellers, W. R.; Zhou, W.; Zhu, P. An F876L mutation in androgen receptor confers genetic and phenotypic resistance to MDV3100 (enzalutamide). Cancer Discovery 2013, 3, 1030.

(5) Hara, T.; Miyazaki, J.; Araki, H.; Yamaoka, M.; Kanzaki, N.; Kusaka, M.; Miyamoto, M. Novel mutations of androgen receptor: a possible mechanism of bicalutamide withdrawal syndrome. Cancer research 2003, 63, 149.

(6) Otsuka, T.; Iguchi, K.; Fukami, K.; Ishii, K.; Usui, S.; Sugimura, Y.; Hirano, K. Androgen receptor W741C and T877A mutations in AIDL cells, an androgen-independent subline of prostate cancer LNCaP cells. Tumor Biol. 2011, 32, 1097.

(7) Tian, X.; He, Y.; Zhou, J. Progress in antiandrogen design targeting hormone binding pocket to circumvent mutation based resistance. Front. Pharmacol. 2015, 6, 57.

(8) Arora, V. K.; Schenkein, E.; Murali, R.; Subudhi, S. K.; Wongvipat, J.; Balbas, M. D.; Shah, N.; Cai, L.; Efstathiou, E.; Logothetis, C.; Zheng, D.; Sawyers, C. L. Glucocorticoid receptor confers resistance to antiandrogens by bypassing androgen receptor blockade. Cell 2013, 155, 1309.

(9) Claessens, F.; Helsen, C.; Prekovic, S.; Van den Broeck, T.; Spans, L.; Van Poppel, H.; Joniau, S. Emerging mechanisms of enzalutamide resistance in prostate cancer. Nat. Rev. Urol. 2014, 11, 712 .

(10) Lee, Y. W.; Chen, T. L.; Shih, Y. R.; Tsai, C. L.; Chang, C. C.; Liang, H. H.; Tseng, S. H.; Chien, S. C.; Wang, C. C. Adjunctive traditional Chinese medicine therapy improves survival in patients with advanced breast cancer: a population-based study. Cancer 2014, 120, 1338.

(11) Ye, L.; Jia, Y.; Ji, K. E.; Sanders, A. J.; Xue, K.; Ji, J.; Mason, M. D.; Jiang, W. G. Traditional Chinese medicine in the prevention and treatment of cancer and cancer metastasis. Oncol. Lett. 2015, 10, 3459.

(12) Bao, J.; Dai, S. M. A Chinese herb Tripterygium wilfordii Hook $\mathrm{F}$ in the treatment of rheumatoid arthritis: mechanism, efficacy, and safety. Rheumatol. Int. 2011, 31, 1123.

(13) Han, R.; Rostami-Yazdi, M.; Gerdes, S.; Mrowietz, U. Triptolide in the treatment of psoriasis and other immune-mediated inflammatory diseases. Br. J. Clin. Pharmacol. 2012, 74, 424.

(14) Wolfram, J.; Suri, K.; Huang, Y.; Molinaro, R.; Borsoi, C.; Scott, B.; Boom, K.; Paolino, D.; Fresta, M.; Wang, J.; Ferrari, M.; Celia, C.; Shen, H. Evaluation of anticancer activity of celastrol liposomes in prostate cancer cells. J. Microencapsulation 2014, 31, 501.

(15) Yang, H.; Chen, D.; Cui, Q. C.; Yuan, X.; Dou, Q. P. Celastrol, a triterpene extracted from the Chinese "Thunder of God Vine," is a potent proteasome inhibitor and suppresses human prostate cancer growth in nude mice. Cancer Res. 2006, 66, 4758.

(16) Zhao, F.; Huang, W.; Zhang, Z.; Mao, L.; Han, Y.; Yan, J.; Lei, M. Triptolide induces protective autophagy through activation of the CaMKKbeta-AMPK signaling pathway in prostate cancer cells. Oncotarget 2015, 6783.

(17) Huang, W.; He, T.; Chai, C.; Yang, Y.; Zheng, Y.; Zhou, P.; Qiao, X.; Zhang, B.; Liu, Z.; Wang, J.; Shi, C.; Lei, L.; Gao, K.; Li, H.; Zhong, S.; Yao, L.; Huang, M. E.; Lei, M. Triptolide inhibits the proliferation of prostate cancer cells and down-regulates SUMOspecific protease 1 expression. PLoS One 2012, 7, e37693.

(18) Li, H.; Zhou, H.; Wang, D.; Qiu, J.; Zhou, Y.; Li, X.; Rosenfeld, M. G.; Ding, S.; Fu, X. D. Versatile pathway-centric approach based on high-throughput sequencing to anticancer drug discovery. Proc. Natl. Acad. Sci. U. S. A. 2012, 109, 4609.

(19) Balbas, M. D.; Evans, M.; Hosfield, D. J. Overcoming mutationbased resistance to antiandrogens with rational drug design. eLife 2013, 2, 499.

(20) Joseph, J. D.; Lu, N.; Qian, J.; Sensintaffar, J.; Shao, G.; Brigham, D.; Moon, M.; Maneval, E. C.; Chen, I.; Darimont, B.; Hager, J. H. A clinically relevant androgen receptor mutation confers resistance to second-generation antiandrogens enzalutamide and ARN-509. Cancer Discovery 2013, 3, 1020.

(21) Lipsky, P. E. D., Tao, X.-L.; Cai, J.; Kovacs, W. J.; Olson, N. J. Selecting substances for treating glucocorticoid-mediated inflammation or immune diseases using Tripterygium wilfordii Hook $F$ extracts. Board of Regents, The University of Texas System (Austin, TX), 1998. 